\title{
Application d'un modèle hydrologique à un bassin versant forestier de Wallonie
}

\author{
F. DEVILLEZ et H. LAUDELOUT \\ Université Catholique de Louvain-la-Neave \\ Centre de Recherches des Ealx et Forits, route de la Fagne, B-6460 Chimay
}

\begin{abstract}
Résumé
Parmi la pléthore de modèles hydrologiques, il n'y en a qu'un petit nombre qui s'adresse aux bassins forestiers. Au sein de ces derniers, il en est qui nécessitent des intrants tels qu'ils ne sont applicables qu'à quelques forêts privilégiées proches de stations agroclimatiques bien équipées. D'autres, évidemment moins précis, se contentent d'un petit nombre d'intormations simples.
\end{abstract}

Le présent article analyse les mécanismes et présente les premiers résultats de l'adaptation à la forêt feuillue européenne du modèle développé par FEDERER et al. (1978. 1979) aux U.S.A. S’il a retenu notre attention, c'est qu'il semblait à première vue conbiner les avantages des deux types.

Le nombre d'intrants se limite aux relevés journaliers de précipitation et de température. Ces données sont utilisées dans une série de sous-routines qui calculent au jour le jour les valeurs d'une série d'indices écoclimatiques classiques dont notamment l'évaporation du sol et du couvert végétal, la transpiration...

Mais ce modèle ne peut tourner que si l'on a préalablement fixé les valeurs d'une série de paramètres caractérisant les éléments géographiques, géologiques, topographiques, pédologiques et phénologiques, susceptibles d'influencer la décomposition du budget des précipitations. Ces paramètres interagissent évidemment avec le climat thermique et hydrique.

L'application de ce modèle à un bassin versant de la forêt de la Fagne de Chimay a consisté, dans une première étape, à l'alimenter avec les données des précipitations et des températures locales (1983 et 1984). Nous n'avons modifié ou adapté que les valeurs des paramètres typiquement propres à notre écosystème, comme la latitude, l'indice foliaire, l'épaisseur de la zone racinaire... Une large part du travail est consacrée aux discussions qui en découlent.

La comparaison des débits ainsi calculés à ceux effectivement mesurés montre que le modèle de FeDERER est apte à prédire avec une précision satisfaisante le débit d'un bassin versant forestier européen. En simulant, par la suite, des fluctuations des paramètres, il est apparu notamment qu'il est relativement peu sensible à des différences importantes des propriétés hydriques des sols. Par contre, il réagit plus rapidement à l'indice foliaire. Ce qui montre que les équations choisies sont particulièrement bien adaptées à un ćcosystème forestier dans lequel la couverture végétale, à la fois complexe et stable, détermine l'évaporation des surfaces en même temps qu'elle conditionne une grande partie du mouvement de l'eau dans les couches superficielles du profil.

Mots clés : Bassin versant, débit, évapotranspiration, hydrologie, modèle, Wallonie. 


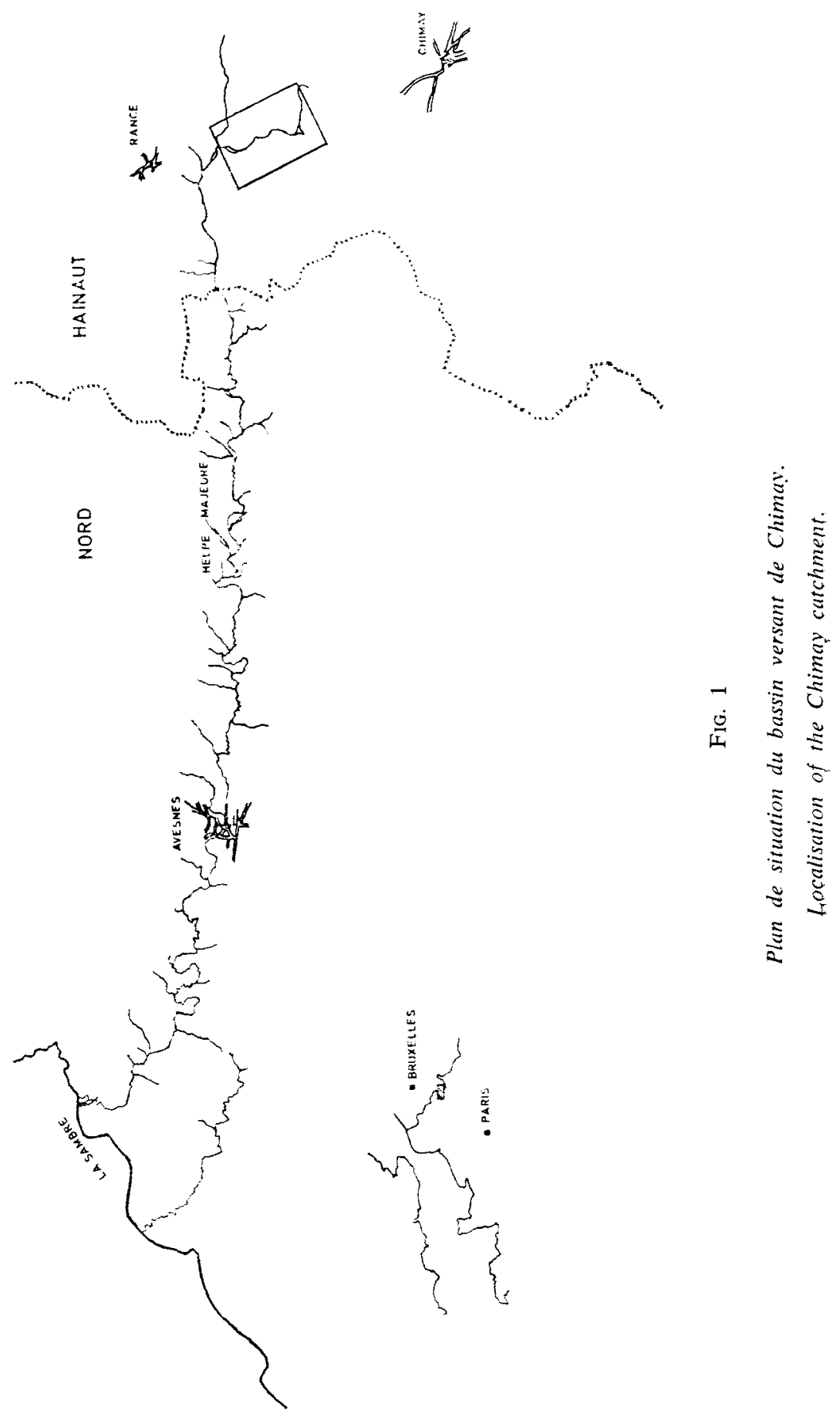




\section{Introduction}

Le calcul du rendement hydrologique d'un bassin versant forestier débouche sur de nombreux aspects pratiques, comme par exemple, la question de prévoir les effets du couvert végétal, de l'aménagement ou de la sylviculture sur la gestion des ressources en eau. Inversement, ce sont les effets des sécheresses estivales sur la productivité des essences indigènes ou exotiques en fonction du type de milieu qui peuvent être recherchées. Les problèmes à résoudre sont d'autant plus intéressants que les sols actuellement encore dévolus aux forêts sont le plus souvent trop secs, trop humides ou caractérisés par un mauvais régime hydrique.

D'un point de vue plus fondamental, l'approche écosystémique de la biogéochimie d'un bassin versant suppose la possibilité de mesurer ou de calculer le débit de ce bassin. Mais, très souvent, il sera impossible d'installer, de maintenir ou d'observer quotidiennenemt les limnigraphes ou déversoirs destinés à enregistrer ou à mesurer les débits des rivières. Il y a donc nécessité de pouvoir calculer jour par jour ce débit avec une fiabilité suffisante à partir d'un modèle mathématique peu exigeant en information et dont la validation soit aisée au moyen d'un minimum de mesures ponctuelles.

Parmi la pléthore de modèles hydrologiques, il n'y en a qu'un petit nombre qui s'adresse aux bassins forestiers. Par ailleurs, le choix d'un modèle ou la nécessité d'en développer un nouveau doit s'inspirer avant tout de l'utilisation envisagée pour les prédictions que l'on peut en tirer. On a répété à satiété que la recherche d'un modèle à toute fin est stérile; il faut en effet se fixer tout d'abord sa finalité, l'information dont on dispose et par conséquent, simplifier l'analyse du problème dans la mesure du but recherché et des moyens disponibles.

Parmi les modèles hydrologiques forestiers proposés au cours de ces quelques dernières années : celui de VAN DER Ploeg (1978) en Allemagne, le modèle de BIRKENES utilisé en Scandinavie notamment par Grip (1982) après sa mise au point dans le Sud de la Norvège par LunDouist $(1976,1977,1978)$ et qui a essentiellement été utilisé pour la distribution du sulfate provenant de pluies acides (CHRISTOPHERSEN \& WRight, 1981), nous avons donné la préférence au modèle «Brook » développé par FEderer et al. $(1978,1979)$ pour les forêts du Hubbard Brook Forest Laboratory. Les raisons en sont à trouver dans son réalisme et sa simplicité, le nombre extrêmement limité des paramètres à ajuster et, enfin, une précision devant satisfaire les gestionnaires de la forêt.

\section{Méthodes}

\subsection{Le site}

1) Le bassin versant étudié (fig. 1) est drainé par un affluent de l'Helpe majeure (Eau d'Eppe localement) : le Rieu d'Ostenne. L'Helpe majeure se jette dans la Sambre un peu en amont de Maubeuge (Nord) après avoir traversé Avesnes-surHelpe (Nord). Tout le bassin $\left(50^{\circ} 06^{\prime}\right.$ de latitude nord et $4^{\circ} 16^{\prime}$ de longitude est) 
est situé à quelque $200 \mathrm{~km}$ au Nord de Paris. L'altitude varie de 210 à un peu plus de 260 mètres.

2) En ce qui concerne le climat régional, les seules données synthétiques relatives au district mosan dont nous disposons sont celles publiées par Poncelet \& MARTIN (1947) pour la période allant de 1911 à 1930 ; elles sont résumées dans le tableau 1.

\section{TABLEAU 1}

Données climatiques applicables au bassin versant de la Fagne de Chimay.

Climatic data for the catchment studied in the Fagne de Chimay.

\begin{tabular}{|c|c|c|c|}
\hline & \multicolumn{2}{|c|}{$\begin{array}{c}\text { PONCILLET \& MARTIN (1947) } \\
1911-1930\end{array}$} & \multirow{2}{*}{$\begin{array}{c}\text { Bassin versant } \\
1981-1984\end{array}$} \\
\hline & District mosan & Forges-Scourmont & \\
\hline Précipitations (en $\mathrm{mm}$ ) $\ldots \ldots$ & $350-1050$ & 1030 & 956 \\
\hline $\begin{array}{c}\text { Précipitations de mai à sep- } \\
\text { tembre }(\text { en } \mathrm{mm})\end{array}$ & $375-450$ & 380 & 466 \\
\hline Maximum de précipitations .. & juillet - décembre & & mai - oct.-déc. \\
\hline Température moyenne annuelle & $7 " 5-9^{\circ}$ & $8 * 3$ & $9 " 6$ \\
\hline $\begin{array}{l}\text { Température moyenne de juil- } \\
\text { let } \ldots \ldots \ldots \ldots \ldots \ldots \ldots \ldots \ldots \ldots . . . . . \\
\text { Température moyenne de jan- }\end{array}$ & $15^{\circ} 5-16^{\circ} 5$ & $16^{\circ} 5$ & $17 " 4$ \\
\hline $\begin{array}{l}\text { Temperature moyenne de jan- } \\
\quad \text { vier } \ldots \ldots \ldots \ldots \ldots \ldots \ldots \ldots \ldots \ldots \\
\text { Nombre moyen de jours de }\end{array}$ & $1^{\prime \prime}-2^{\prime \prime}$ & $0 \% 6$ & $2 " 5$ \\
\hline végétation $\ldots \ldots \ldots \ldots \ldots$ & $150-200$ & 160 & 193 \\
\hline $\begin{array}{c}\text { Nombre moyen de jours de } \\
\text { gelée } \ldots \ldots \ldots \ldots \ldots \ldots \ldots\end{array}$ & $80-100$ & $80-100$ & 70 \\
\hline Date des dernières gelées ... & $10-20 \mathrm{mai}$ & $6 \mathrm{mai}$ & $10 \mathrm{mai}$ \\
\hline
\end{tabular}

Ce dernier reprend aussi à titre de comparaison, pour la même période, les résultats des observations faites à Forges-Scourmont, station climatique la plus proche de notre bassin versant à cette époque. Celle-ci se trouve déjà en Ardenne et est donc représentative d'un climat plus froid et plus humide qu'à Chimay. De par leur situation, les écosystèmes étudiés sont soumis, sans nul doute, à un climat intermédiaire entre celui du district mosan et celui de la Basse-Ardenne. Enfin, la troisième colonne du tableau 1 traduit le climat moyen du bassin versant pour la période 1981-1984 au départ des observations locales.

3) A part les alluvions modernes des fonds de vallée associées à des dépôts limoneux des pentes, la lithologie est dominée par le Famennien inférieur avec l'Assise de Senzeilles surtout et celles de Mariembourg et d'Esneux. Le Famennien supérieur est représenté par l'Assise de Souverain-Pré dans le sud du bassin versant.

Les sols développés sur ces assises du Famennien sont de type argileux compact à mauvais régime hydrique. Mais on observe aussi des dépôts lœssiques d'argile ou de limon, surtout sur les plateaux. Les seconds donnent en principe des sols moins 

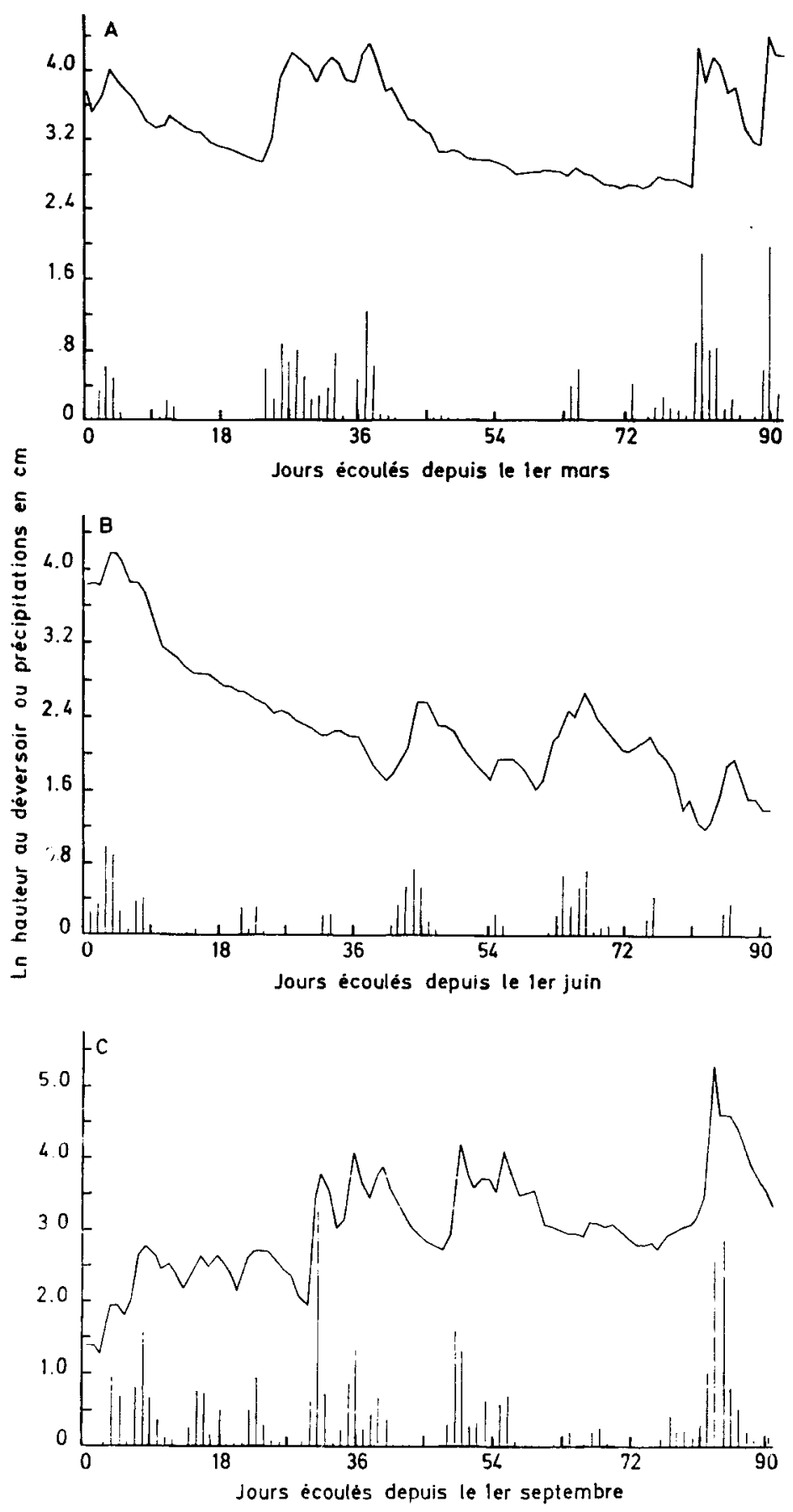

Fig. 2

Evolution au cours de l'année 1984 du coefficient de tarissement du bassin versant de Chimay. Il est exprimé par la variation du logarithme népérien de la hauteur au déversoir (-) avec le temps.

Evolution for 1984 of the recession of the Chimay catchment. It is expressed by the change of the natural logarithm of the weir height with time. 
lourds que ceux dérivant de la décomposition des schistes. Mais de faible épaisseur, ils reposent le plus souvent sur un substrat mal drainé, comportent un horizon B textural ou sont gleyifiés à faible profondeur (REмY, 1980, 1981).

La conséquence de la nature du substrat géologique est que les réactions du débit du bassin aux précipitations sont rapides comme le montre la figure 2 dans laquelle on a placé en abcisse le temps et en ordonnée les précipitations ou le ln de la hauteur lue au déversoir : soit une grandeur proportionnelle au débit $\mathrm{Q}$ à la puissance 2,5 .

Le coefficient de tarissement $k$ peut être exprimé par la relation :

où $\mathrm{t}$ est le temps en jours.

$$
\mathrm{Q}=\mathrm{Q}_{0} \exp (-\mathrm{kt})
$$

Le calcul de 7 courbes de récession pendant les trois mois d'élé a donné une valeur moyenne de $0,42 \pm 0,14$ jour $^{-1}$ pour la valeur $k$, soit 1.6 jour pour réduire $Q_{0}$ à la moitié de sa valeur.

Lorsque la sécheresse se prolonge, le tarissement se fait à un rythme plus lent,

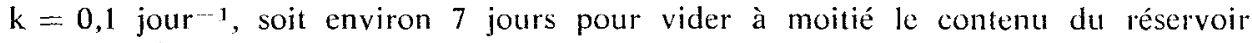
correspondant.

Ces valeurs correspondent à peu près à ce qui a été mesuré par Monjore \& Cajot (1983) qui trouvent des coefficients de tarissement superficiel de 0,6 à 1,5 jour 1 suivis par des coefficients de tarissement allant de 0,1 à 0,3 jour 1 .

4) Le taux de boisement du bassin versant est de l'ordre de 95 p. 100 ; le reste est occupé par des prairies permanentes et des étangs de pêche. Les plantations résineuses, relativement jeunes, sont de peu d'importance. Quelques aulnaies se développent sur les sols constamment engorgés. Ailleurs, c'est la chênaie-charmaic qui occupe le terrain.

Cette forêt feuillue est exploitée depuis des siècles en taillis-sous-futaie; mais, en bon nombre d'endroits, ce dernier est en voie de conversion en futaie. Elle correspond donc à un groupement de substitution de la forêt climatique primitive que l'on peut rattacher (LAudelout el al., 1984) au Pulmonario-Carpinetum (Sougnez, 1967).

\subsection{La structure du modèle}

La structure du modèle hydrologique utilisé a été présentée en détail dans une publication relativement peu accessible en Europe (FEDkrer \& LASH, 1978). Les exposés qui en ont été faits dans d'autres publications de FEDErer sont nettement moins détaillés et il nous a semblé utile d'en reproduire ici les traits essentiels.

Le principe de base ne diffère pas de celui qui est adopté dans tous les modèles hydrologiques : il y a conservation de la quantité d'eau et par conséquent, les entrées d'eau dans le bassin versant moins les sorties sont égales à la variation du stock d'eau dans le bassin.

Le modèle distribue l'eau dans 5 compartiments :

1) l'eau dans la zone racinaire; 
2) l'eau dans le sol non saturé au-dessus du niveau de la nappe mais en dessous de la zone 1 ;

3) la nappe phréatique ;

4) la neige interceptée par la végétation forestière:

5) la neige sur le sol.

Cette approche par l'analyse "compartimentale" est intermédiaire entre celle du modèle purement numérique (boîte noire) et celle du modèle strictement déterministe. On verra que certains des chaînons du modèle sont purement déterministes (interception, évaporation), d'autres le sont nettement moins (mouvement de l'eau dans la zone non saturée).

Le schéma de la figure 3 représente les divers compartiments et les flux qui les connectent. On voit que la zone racinaire a été subdivisée en deux sous-compartiments : l'un, qui évapore directement; l'autre, qui ne participe qu’à la transpiration.

Les précipitations sont le seul flux d'entrée, tandis que l'évapotranspiration, l'infiltration profonde et le débit de la rivière sont les flux de sortic.

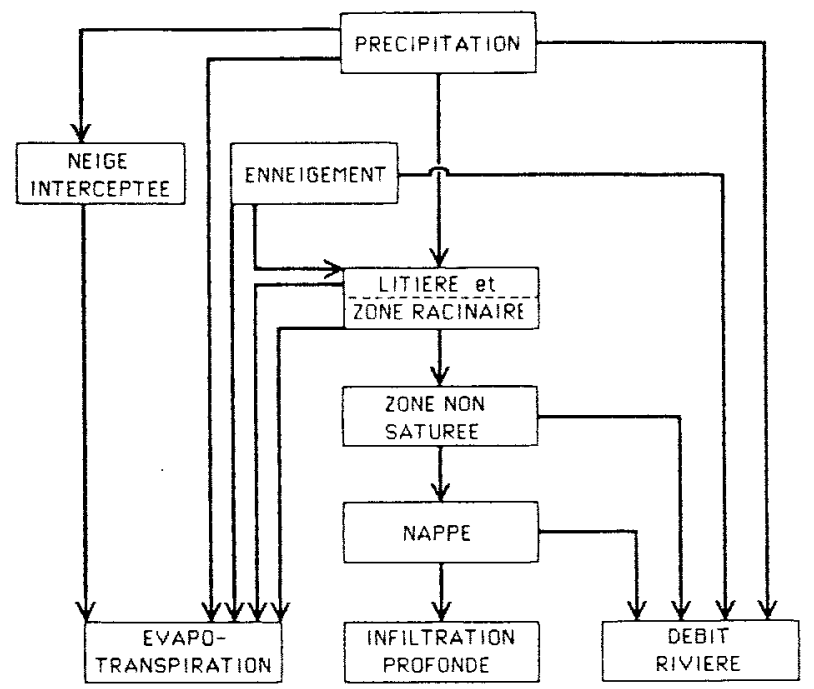

FIG. 3

Schéma de distribution de l'eau entre les divers compartiments définis par le modele «BROOK».

Les flèches représentent les flux d'un compartiment à l'autre.

Distribution scheme of water in the various compartments of the «BROOK» moted.

The arrows show the flow from a compartment to another.

Les contenus des divers compartiments sont exprimés en mm. Le modèle intègre les flux (en $\mathrm{mm}$ ) par différences finies sur un intervalle de temps fixe $\Delta t$ qui sera toujours égal à 1 jour. On aura ainsi : 
stock d'eau au temps $t+\Delta t=$

stock d'eau au temps $t+$ (Flux d'entrée - Flux de sortie) $\Delta t$

Nous présenterons les principales équations utilisées comme elles sont écrites dans le modèle, c'est-à-dire suivant la syntaxe du FORTRAN.

\subsection{Les mesures}

1) Il convient d'abord de réaliser une estimation aussi précise que possible de la quantité d'eau qui est tombée sur le bassin de façon à établir un des termes du bilan d'eau qui est à la base de tout modèle hydrologique. Cependant, comme le démontre encore une étude récente (LANDSBERG, 1983), la variabilité spatiale est considérable.

Nous avons été obligés de nous limiter à un réseau de 6 pluviomètres dont deux enregistrcurs situés : l'un à $7 \mathrm{~km}$ at nord du bassin versant (Sivry CPEN) à la même altitude; l'atitre à $4 \mathrm{~km}$ au sud du bassin (Bailièvre). Quatre pluviomètres étaient situés dans les clairières plus ou moins étendues situées à l'intérieur du bassin. Aucune tendance systématique à la variation suivant une direction N.S. ou E.W. ne s'est manifestée. Comme le montre la figure 4, les précipitations cumulées par pentade (pour 1983 et 1984), pour les deux stations extrêmes (Sivry et Bailièvre) situées à la même altitude à $11 \mathrm{~km}$ l'une de l'autre sont suffisamment homogènes.

Nous avons donc utilisé comme intrant la moyenne des totaux journaliers des six postes pluviométriques. Les précipitations sous forme de brouillard ou de rosée sont négligées.

2) Les températures moyennes journalières ont été calculées à partir des températures minimales et maximales mesurées sous abri à Sivry. Ces températures interviennent dans le calcul de l'évapotranspiration et pour départager les pluies des neiges (voir D).

3) Les débits de la rivière drainant le bassin ont été mesurés de façon ponctuelle en 1980,1981 et 1982 par la méthode de dilution intégrale; puis, en 1983 et 1984, de façon journalière, par un déversoir à paroi mince de 60" d'angle d'ouverture installé dans un caniveau en béton. Comme la mise en place de ce déversoir, constilué d'une plaque d'aluminium boulonnée dans l'ouverture amont du caniveau, n'était certes pas idéale, nous l'avons calibré en utilisant la méthode de dilution intégrale. L'équation théorique pour la relation entre le côté mouillé du déversoir et le débit en litres par minute est :

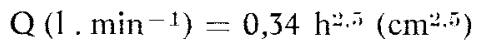

La relation trouvée expérimentalement par la méthode de dilution intégrale était suffisamment proche, soit :

$$
\mathrm{Q}=0,33 h^{2.5}
$$

Les résultats donnés par la méthode de dilution intégrale ont élé utilisés pour le calcul du coefficient de dispersion longitudinale et de la fraction d'eau morte, ce qui permettait le calcul de débits extrêmement faibles. Les détails sur cette méthode sont présentés dans un article récent (Legrand-Marco \& LaUdelout, 1985). 

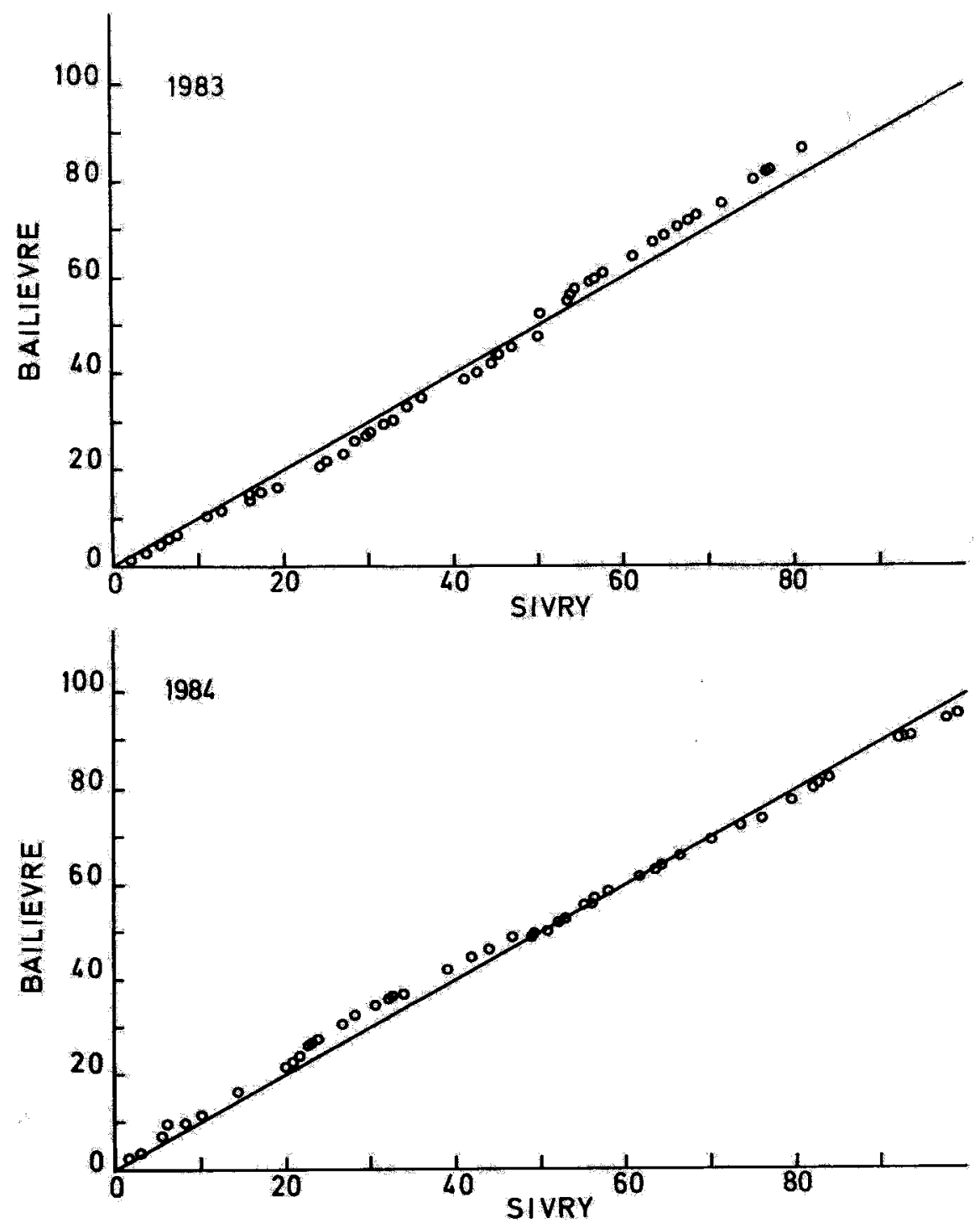

FrG. 4

Comparaisons des prếcipitations en 1983 et 1984 (en cm par pentades) pour les stations de Sivry et Bailevere.

Comparison of the stations of Sivry and Bailieve for 1983 and 1984 rainfalls (in em per 5 day periodst. 


\subsection{L'ajustement des paranètres}

Outre son alimentation en valeurs journalières de température et de précipitations, le modèle hydrologique "BROOK » exige que soient fixées les valeurs d'une série de paramètres. Le tableau 2 résume pour chacun de ceux-ci les valeurs utilisées et leurs dimensions.

On remarquera que lorsque les valeurs des paramètres déterminés pour les forêts da New Hampshire et de la Caroline du Nord sont suffisamment voisines, nous en avons pris la moyenne pour l'application à Chimay. Lorsqu'elles sont très différentes (par exemple, celle du paramètre régissant l'évaporation par la litière), nous avons préféré choisir celle du New Hampshire dont le type de forêt est plus proche de celui de Chimay. La différence essentielle entre la forêt de la Caroline du Nord et celle qui nous occupe semble être la présence d'une strate herbacée ct arbustive sempervirente qui doit modifier considérablement l'évaporation de la couverture morte pendant les mois d'hiver.

Nous commenterons ci-dessous les paramètres qui le méritent ainsi que les modifications apportées pour les adapter aux conditions de la Fagne de Chimay.

\subsection{L'interception de la plaie par le couvert}

a) Dans un écosystème forestier, l'interception de l'eau par le couvert végétal et sa réévaporation après la pluie constituent un des éléments essentiels pour estimer le rendement en eau des précipitations. En effet, de la décomposition du budget des précipitations, il ressort que :

avec :

$$
E=P-\left(E_{c}+P N+S\right)
$$

E : évaporation des surfaces développées par les arbres;

$P$ : précipitations incidentes au peuplement;

$\mathbf{E}_{c}$ : eau qui s'écoule le long des troncs;

$\mathrm{PN}$ : égouttement $=\mathrm{T}+\mathrm{D}$;

$T$ : précipitations atteignant directement le sol:

D : précipitations arrêtées par les cimes et qui tombent au sol par la suite;

$\mathbf{S}$ : eau absorbée par les parties aériennes des arbres.

On a également :

où INT est la pluie interceptée.

$$
\mathrm{INT}=\mathrm{E}+\mathrm{S}
$$

Comme le terme $\mathrm{S}$ est négligeable, il ressort que l'importance de l'évaporation est inversement proportionnelle à la fraction des précipitations atteignant directement ou indirectement le sol.

b) Le modèle hydrologique «BROOK» utilise comme paramètre d'estimation de linterception de la pluie ou de la neige, l'indice foliaire (LAI : leaf area index) et l'indice de lige (SAI : stem area index) des strates arbustives et arborescentes. 


\section{Tableau 2}

Paramètres utilisés dans les calculs pour trois sifes.

Parameters used at three sites.

\begin{tabular}{|c|c|c|c|}
\hline Paramètre & $\begin{array}{l}\text { Hubbard } \\
\text { Brook } \\
\text { N.H. }\end{array}$ & $\begin{array}{l}\text { Coweeta } \\
\text { N.C. }\end{array}$ & Chimay \\
\hline Latitude ("N) & $43^{\prime \prime} 95$ & $35^{\prime \prime} 05$ & $50^{\circ} 0$ \\
\hline Pente $(\%) \ldots$ & 12,1 & 18,0 & 2.0 \\
\hline Orientation (") $\ldots \ldots \ldots \ldots \ldots \ldots$ & $203^{\circ} 2$ & $18^{\circ} 0$ & $300^{\circ}$ \\
\hline Coefficient d'interception (Equ. 5) ... & 0,75 & 0,75 & 0,75 \\
\hline $\begin{array}{l}\text { Température de séparation pluie-neige } \\
\left({ }^{\circ} \mathrm{C}\right) \quad \ldots \ldots \ldots \ldots \ldots \ldots \ldots \ldots \ldots\end{array}$ & -2.8 & $0^{\prime \prime} 0$ & $-0 " 4$ \\
\hline LAI (indice foliaire) $\ldots \ldots \ldots \ldots \ldots$ & 4,0 & 4,0 & 4,0 \\
\hline SAI (indice surface troncs) $\ldots \ldots \ldots$ & 2,0 & 2,0 & 2,0 \\
\hline Dates de début de la feuillaison ..... & 136 à 166 & 106 a 136 & 116 à 152 \\
\hline $\begin{array}{l}\text { Dates de fin de la défeuillaison ..... } \\
\text { Parametres de calcul des sources dircetes }\end{array}$ & $258 \grave{\text { à }} 288$ & 289 à 321 & $237 \grave{a} 314$ \\
\hline $\begin{array}{l}\text { Fraction imperméable de la surface du } \\
\text { bassin (IMPERV dans Equ. } 8 \text { ) } \ldots . .\end{array}$ & 0,01 & 0,01 & 0,01 \\
\hline $\begin{array}{l}\text { Coefficient du taux d'humidité dans } \\
\text { l'exponenticlle (PAC, Equ. 6) } \ldots . .\end{array}$ & 40,0 & 25,0 & 40,0 \\
\hline $\begin{array}{l}\text { Facteur de l'exponentielle (PC, Equ, 6) } \\
\text { Caractéristiques du profil }\end{array}$ & $0,41.10^{\cdots}$ & $0,74.10$ & $0.41 .10^{-\bar{z}}$ \\
\hline Epaisseur de la couche racinaire $(\mathrm{m})$.. & 0,64 & 0,90 & 0,31 \\
\hline Epaisscur de la litière $(\mathrm{m}) \ldots \ldots \ldots \ldots$ & 0,05 & 0,05 & 0,05 \\
\hline $\begin{array}{l}\text { Epaisseur de la zone non saturée }(\mathrm{m}) \\
\text { Coefficient de la relation } \mathrm{k}(0) \text { : } \\
\text { Facteur KEINT et KUINT des equations }\end{array}$ & 0,04 & 4,20 & 004 \\
\hline $\begin{array}{c}10 \text { et } 11 \ldots \ldots \ldots \ldots \ldots \ldots \\
\text { Exposants KUSLP de l'équation } 10 \text { et } 11\end{array}$ & $\begin{array}{c}2,04.10^{\circ} \\
12,56\end{array}$ & $\begin{array}{c}1,05.10 \\
11,74\end{array}$ & $\begin{array}{l}1,50.10^{-} \\
12,00\end{array}$ \\
\hline Paramètres déterminant IETR & & & \\
\hline $\begin{array}{l}\text { Paramètre déterminant la disponibilité } \\
\text { de l'eau du sol pour la transpiration } \\
\text { (CT, Equ. I6) } \ldots \ldots \ldots \ldots \ldots \ldots \ldots \ldots\end{array}$ & 28 & 25 & 28 \\
\hline $\begin{array}{l}\text { Teneur en eau à une tension de }-15 \text { bar } \\
\text { Paramètre déterminant la disponibilité de }\end{array}$ & 0,09 & 0,09 & 0,09 \\
\hline $\begin{array}{l}\text { l'eau du sol pour l'évaporation par la } \\
\text { litière }(C E) \quad \ldots \ldots \ldots \ldots \ldots \ldots \ldots \ldots\end{array}$ & 12 & 3 & 12 \\
\hline $\begin{array}{l}\text { Coefficient multiplicatif de l'ETP cal- } \\
\text { culée suivant Hamon (PEC) } \ldots \ldots \ldots\end{array}$ & 1 & 1,2 & 1,1 \\
\hline
\end{tabular}


Le LAI se définit comme la surface foliaire (une seule face) développée par unité de surface de sol. Un assez grand nombre de mesures ont été réalisées à proximité immédiate du site étudié sur une végétation très similaire par ScHNock (1967) et SchNock \& Galoux (1960). Nous avons admis une valeur de 4 pour le LAI en accord approximatif avec les valeurs trouvées à Virelles par les auteurs cités.

En ce qui concerne le SAI, nous avons adopté la valeur 2, conformément aux observations de WhitTAKER et al. (1974) sur les forêts feuillues du Hubbard Brook et les observations de SchNock \& Galoux (1960) à Virelles.

L'équation qui décrit l'interception de la pluie a été formulée comme suit par Federer \& Lash (1978) :

$$
\mathrm{INT}=\mathrm{INC} \times(0,666 * \mathrm{LAI} / 4+0,333 * \mathrm{SAI} / 2,0) * \mathrm{MIN}(\mathrm{ETP}, \text { PLUIE })
$$

où INT est la pluie interceptée et MIN la fonction FORTRAN qui prend la plus petite des deux valeurs : PLUIE ou ETP (évaporation potentielle), ETP étant calculé suivant les équations (13), (14) et (15) ci-dessous. Le rôle de la fonction MIN étant d'avoir une interception proportionnelle à la pluie tombée mais ne l'excédant jamais. L'interception est done le fait du LAI pour les $2 / 3$ et du SAI pour 1/3, lorsque l'on ramène l'un et l'autre à l'unité de surface développée.

Le coefficient INC peut se calculer à partir de l'interception mesurée et des valeurs adoptées par LAI et SAI. Nous avons adopté la valeur 0,75 , ce qui permet le calcul d'une interception annuelle moyenne de $147 \mathrm{~mm}$ sur 4 années d'observation, soit 15 p. 100 des précipitations. Cette valeur se place au centre de la fourchette des pourcentages d'interception ( 6 à $30 \mathrm{p}$. 100) fournis par de nombreux auteurs à propos des forêts feuillues du continent : Bodeux, 1954 ; Nolrfalise, 1959 ; Eidmann, 1959 ; Brechtel, 1969; Mitscherlich \& Moll, 1970 ; Molchanov, 1971. Dans les observations plus récentes, elle est très proche des interceptions annuelles moyennes (14 à 18 p. 100) observées dans les peuplements mûrs et de densité normale : AusSEnAC, 1968 ; Bultot et al., 1972 ; Aussenac \& Boulangeat, 1980.

Enfin, l'interception moyenne annuelle observée ici est tout à fait comparablo à celles citées par les auteurs américains : $12 \mathrm{p}$. 100 de la pluie pour la forêt du Hubbard Brook dans le New Hampshire, soit $110 \mathrm{~mm}$ par an (LEONARD, 1961); 13 p. 100 de la pluie, soit $250 \mathrm{~mm}$ à Coweeta en Caroline du Nord (Helvey \& Patric, 1965).

Par contre, dans un site ne se trouvant qu'à une dizaine de $\mathrm{km}$ de notre bassin versant, donc dans des conditions générales quasi identiques, des mesures directes de SchNock \& GALOUX (1967) ont donné une interception de $267 \mathrm{~mm}$ sur une pluviométrie totale de $1159 \mathrm{~mm}$, soit 23 p. 100.

c) En forêt de feuillus, il est tout à fait logique que le taux d'interception varie considérablement au cours des saisons : selon les auteurs, dans des rapports allant de $3 / 1$ à $4 / 3$ entre la phase feuillée et la phase défeuillée. Mais, dans la majorité des cas, on admet qu'en période de feuillaison, l'interception est le triple de ce qu'elle est en phénophase défeuillée. Helvey \& Patric (1965) et Léonard (1961) avaient trouvé une interception de plus des deux tiers en phase défeuillée qu'en phase feuillée. Ces observations américaines recoupent celles de ScHNock \&. GaLOux (1967) qui ont trouvé pour deux années successives des interceptions de 34 et 27,1 en phénophase feuillée contre 27 et 20 en phénophase défeuillée; ce qui représente 
effectivement plus des deux tiers, quelle que soit la valeur des taux dinterception mesurés que nous avons discutés ci-dessus.

Le SAI peut évidemment être considéré comme constant au cours de l'année en l'absence d'exploitation drastique ou de mise à blanc. Par contre, c'est la variation annuelle du LAI qui est responsable de l'évolution correspondante de l'interception en forêt feuillue; celle-ci n'intervient pas sans modification d'une année sur l'autre. La difficulté essentielle consiste donc à introduire dans les données du programme les dates clés. Nous avons dû nous contenter provisoirement des valeurs suivantes qui correspondaient à peu près aux observations de 1984 à Chimay :

- début de la feuillaison : 25 avril, soit jour 116 ;

- fin de la feuillaison : $1^{\text {rr }}$ juin, soit jour 152 ;

- début de la défeuillaison : 25 août, soit jour 237 ;

- fin de la défeuillaison : 10 novembre, soit jour 314.

On admet que la feuillaison progresse ou régresse de façon linéaire entre les dates extrêmes qui ont été citées.

\subsection{L'enneigement}

Le modèle «BROOK» élaboré par FEDERER consacre beaucoup de temps aux problèmes de l'interception de la neige, de son évaporation, de sa fonte, etc. Nous ne nous sommes guère préoccupés ici de l'ajustement des paramètres, étant donné que l'enneigement de l'écosystème forestier que nous avons étudié se réduit à très peu de chose en comparaison à l'enneigement des forêts du New Hampshire qui dure de novembre à avril.

Le modèle n'accepte comme intrant que les précipitations quelles que soient leur forme, il comporte donc une formulation qui permet de séparer les précipitations en pluie ou neige. Cette opération est réalisée en transformant la pluie en neige entre deux valeurs extrêmes de température ou plus simplement encore en admettant que toute précipitation pour une température inférieure à une valeur donnée tombe sous forme de neige et détermine un enneigement du sol et une interception par le couvert dont le modèle calcule la durée.

Pour le climat du New Hampshire, c'est la température de $-2,4$ "C qui constitue ce seuil. Dans les conditions climatiques de la Fagne de Chimay, nous avons observé que la température de $-0,4^{\circ} \mathrm{C}$ était plus appropriée. Nous nous sommes limités à cet ajustement du modèle en raison de l'intérêt mineur que présentait une étude approfondie de l'enneigement sous nos conditions. Les chutes de neige et la durée de l'enneigement ainsi calculées ont reproduit de façon à peu près correcte la situation réelle observée pendant quatre hivers.

\subsection{Le débit}

La formation du débit de la rivière drainant le bassin versant peut avoir diverses origines qu'il est bien difficile de séparer dans l'état actuel de nos connaissances en hydrologie.

a) La contribution de la zone saturée au débit de la rivière est supposée nulle. C'est là une condition essentielle pour que le bassin puisse être étudié facilement au point de vue de la constitution biogéochimique des eaux de drainage. 
b) Une fraction de la surface du bassin délivre l'eau des précipitations immédiatement ou quasi immédiatement au système de drainage. Cette fraction « variable source " suivant HEw LETT \& HibBERT (1967) serait générée dans les surfaces saturées au point de vue hydrique des replats alluviaux. Ces surfaces auront une aire variable suivant la sécheresse. La formulation adoptée par FEDERER \& LASH (1978) est la suivante :

$$
\mathrm{PRT}=I M P E R V+\mathrm{PC} * \mathrm{EXP}(\mathrm{PAC} * \mathrm{EZONE} / \mathrm{EZDEP})
$$

où :

PRT est la fraction du bassin fonctionnant comme source;

IMPERV est la fraction de la surface du bassin qui est constamment imperméable plus celle qui correspond à la surface d'eau libre;

EZONE est la hauteur d'eau présente dans la zone racinaire;

EZDEP est la profondeur de la zone racinaire;

les coefficients PC et PAC sont des coefficients qui sont manipulés pour ajuster le débit calculé au débit observé.

En utilisant 6 années d'observations dans les deux bassins étudiés : l'un, dans le New Hampshire, l'autre, en Caroline du Nord, Federer \& Lash (1978) ont obtenu des valeurs de $\mathrm{PC}=4,1 \cdot 10^{- \text {"i }}$ et $\mathrm{PAC}=40$ au Hubbard Brook Forest Laboratory, et $\mathrm{PC}=7,4 \cdot 10^{-5}$ et $\mathrm{PAC}=25$ au Coweeta Watershed.

Cette formulation n'est guère satisfaisante, mais elle reflète l'état insuffisant de nos connaissances en hydrologie auquel il a été fait allusion plus haut. Pour des raisons évidentes (analogie de végétation et de climat), nous avons adopté les valeurs du Hubbard Brook Laboratory pour Chimay.

c) Le transfert de l'eau dans la zone racinaire a été modélisé de façon très simple. Pour l'écoulement suivant les pentes d'un bassin versant, la modélisation aurait nécessité une complexité de calcul en rapport avec la non-linéarité de l'équation aux dérivées partielles et la difficulté d'obtenir une idée précise de la répartition spatiale des propriétés de conductivité hydraulique; on serait alors arrivé rapidement à des programmes d'une lourdeur telle qu'ils ne peuvent guère s'intégrer dans un modèle hydrologique forestier où les données nécessaires doivent être réduites au maximum.

A l'opposé, certains modèles hydrologiques se limitent à considérer une capacité au champ telle que, une fois cette teneur en eau atteinte, le drainage est complet et immédiat et en-deçà de laquelle, il n'y a aucun déplacement d'eau.

Le modèle «BROOK» introduit une simplification de la situation réelle que l'on peut considérer comme intermédiaire entre ces deux extrêmes.

Si le sol de l'horizon racinaire est homogène et n'est pas influencé par la nappe phréatique et que l'on néglige en outre le gradient de potentiel matriciel, de même que les différences d'activité du système racinaire en fonction de la profondeur, on peut se limiter à exprimer le flux d'eau à travers le sol $Q$ sous un gradient unitaire de la charge hydraulique par :

$$
\mathrm{Q}=\mathrm{K}
$$

où $\mathrm{K}$ est la conductivité hydraulique à la teneur moyenne en eau du sol. 
Ceci ne veut évidemment pas dire que la conductivité hydraulique est constante et indépendante de 0 , la teneur en eau du sol. On admet au contraire une relation $K(0)$ telle que celle qui a été "démontrée » par Camprell (1974) :

$$
\mathrm{K}=\mathrm{d}(0)-2 n+\ddot{3})
$$

La constante $b$ est celle que l'on obtient à partir de la relation empirique bien connue pour la courbe de $\mathrm{pF}$ soit :

où $\psi$ 'est le potentiel matriciel.

$$
\psi^{\prime}=c 0^{b}
$$

Si l'on définit la capacité au champ comme la teneur en eau qui correspond à une conductivité hydraulique de $2 \mathrm{~mm}$. jour-1, laquelle sera associée à un potentiel caractéristique du sol, ce potentiel est celui qui correspondra à une humectation complète des couches superficielles, sans bien sûr arriver à la saturation. Connaissant $\mathrm{K}, \theta$ et $\mathrm{b}$, on peut évaluer $\mathrm{d}$.

Les déterminations de Federer et Lash sur deux bassins forestiers ont donné des valeurs assez semblables, soit :

$$
\begin{aligned}
\mathrm{d}= & 2,04 \cdot 10^{7} \text { ou } 1,05 \cdot 10^{7} \mathrm{~mm} \cdot \text { jour }^{-1} \\
& -2 \mathrm{~b}+3=12,56 \text { ou } 11,74
\end{aligned}
$$

Comme il s'agissait dans les deux cas d'horizons humifères forestiers similaires à ccux du bassin étudié, nous avons préféré adopter ces valeurs arrondies, soit $1,5 \cdot 10^{7}$ et 12 plutôt que de préciser la relation $K(0)$. Rappelons que puisque 0 représente l'humidité volumique et est donc constamment inférieure à l'unité, la relation (8) est une exponentielle décroissante pour $-2 b+3>0$.

d) Le flux d'eau de la zone racinaire vers la zone immédiatement sous-jacente pourra donc s'écrire par :

$$
\text { EDRAIN }=\text { KEINT } *(\text { EZONE/ESDEP }) * * \text { KESLP }
$$

avec $\mathrm{d}=$ KEINT, $-2 \mathrm{~b}+3=$ KESLP.

De même, le flux d'eau à la sortie de cette zone non saturée s'exprimera par :

$$
\text { UZOUT }=\text { KUNT } * \text { UZONE/UZDEP) } * * \text { KUSLP }
$$

dont les variables ont un sens analogue à celles de l'équation (10) avec KUINT $=\mathrm{d}$ pour la couche non saturée ; UZONE et UZDEP représentent respectivement la teneur en eau et l'épaisseur de la zone non saturée et KUSLP est l'analogue de $-2 b+3$ pour cette zone.

Pour plus de simplicité, on a pris :

$$
\text { KUINT }=\text { KEINT et KUSLP }=\text { KESLP }
$$

Comme le montre le schéma général, le flux d'eau correspondant au drainage est dirigé immédiatement vers la rivière.

Le schéma qui vient d'être décrit peut sembler assez surprenant; en réalité, il doit être replacé dans le cadre du schéma général du modèle hydrologique lequel 
s'identifie avec une analyse compartimentale du système plutôt qu'à une modélisation sensu stricto. On définit un compartiment contenant de l'eau et l'on tente de calculer le flux d'eau qui en sort. Ce flux est estimé en se servant des lois régissant le mouvement de l'eau dans le sol, mais en faisant abstraction, par exemple, de l'hétérogénéité de la distribution du système racinaire et de son action sur l'évapotranspiration, puisque l'hypothèse fondamentale de l'analyse compartimentale est qu'un compartiment est supposé être homogène. En outre, on néglige les effets d'hystérésis dans les phénomènes d'humectation et de dessication.

Une seconde remarque qui peut être faite au sujet du mode de calcul utilisé est que l'incrément de temps égal à un jour partout dans le modèle peut être la cause d'erreurs de calcul s'il est utilisé pour le mouvement de l'eau à travers le sol non saturé. La raison en est évidemment la variation rapide de $K(\theta)$ avec 0 pouvant conduire à des différences considérables de débit immédiatement après les averses. Aussi, le modèle utilise une sous-routine de calcul pour le mouvement de l'eau dans la zone saturée dans laquelle l'intervalle d'intégration est réduit à un demi-jour ou moins suivant la nécessité.

\subsection{L'évapotranspiration}

a) L'équation choisie pour le calcul de l'évapotranspiration potentielle (ETP) est celle de Hamon (1963); soit en mm - jour ' :

$$
\text { ETP }=0,1651 * \text { JOUR * RHOSAT }
$$

où JOUR est la longueur du jour du lever au coucher du soleil, et RHOSAT, la masse volumique de la vapeur d'eau à la pression de saturation en $\mathrm{g} / \mathrm{m}$ : à la température moyenne journalière TEMP, soit :

$$
\begin{gathered}
\text { RHOSAT }=216.7 * \text { ESAT/(TEMP }+273,3) \\
\operatorname{ESAT}=6,108 * \text { EXP }\{17,269 * \text { TEMP } /(\text { TEMP }+273,3)\}
\end{gathered}
$$

avec ESAT = la tension saturante de la vapeur d'eau à la température TEMP.

Le facteur PEC est un coefficient empirique ajusté pour la validation du modèle par la comparaison des observations et des valeurs calculées et que l'on utilise comme multiplicateur de l'évaporation calculée comme ci-dessus.

Le modèle contient un algorithme qui permet de calculer la longueur du jour en fonction de la latitude et de corriger l'insolation potentielle en fonction de la pente et de l'orientation du bassin. Cet algorithme est celui qui a été proposé par SWIFT (1976).

b) Il est évidemment indispensable de considérer en quelle mesure l'évapotranspiration potentielle qui a été calculée par l'équation (13) est effectivement réalisée pour donner l'évapotranspiration réelle (ETR) dans des circonstances données.

11 existe de nombreuses méthodes pour calculer la réduction que subil l'ETP lorsque l'approvisionnement en eau n'est pas illimité. Celle qui est choisic dans le modèle consiste à considérer que l'évapotranspiration réelle sera égale soit à l'ETP, soit à une fonction de l'état hydrique du sol. 
On aura :

ou

$$
\mathrm{ETR}=\mathrm{ETP} \text {, si } \theta_{\mathrm{tim} \text { yonithe }}>\mathrm{CT} * \mathrm{ETP}
$$

$$
\mathrm{ETR}=\frac{\theta_{\text {disponitite }}}{\mathrm{CT}} \text {, si } 0_{\text {inis } \text { onible }}<\mathrm{CT} * \mathrm{ETP}
$$

Dans ces deux équations :

$$
\theta_{\text {disponible }}=\theta-\theta_{15}
$$

où $\theta_{15}$ est la teneur en eau à une tension de -15 bar, soit le point de fanaison permanent. Ce point est fourni par la courbe de $\mathrm{pF}$ du sol et correspond à $\theta=0,09$. La constante CT est empirique. Elle a toutefois un sens physique puisqu'elle peut être considérée comme l'inverse de la pente de la relation (supposée linéaire) entre la teneur en eau du sol et l'évapotranspiration. Comme l'évapotranspiration est calculée en $\mathrm{mm} \cdot$ jour $^{-1}$ et la teneur en eau du sol en $\mathrm{mm}$, la constante aura les dimensions jour.

Les valeurs calculées pour CT dans les deux bassins versants forestiers américains étaient 28 et 25 jours basées sur près de 20 ans d'observation. Nous avons utilisé la valeur de 27 jours. Comme on le verra ci-dessous, on utilise un procédé analogue pour le calcul de l'évaporation par la litière (ou de l'évapotranspiration par la couverture herbacée). Le paramètre $\mathrm{CE}$ du tableau 2 y joute le même rôle que CT. Les valeurs trouvées pour les deux bassins forestiers américains étant très différentes, nous avons préféré utiliser celle du New Hampshire.

L'évolution saisonnière de l'évapotranspiration est évidemment affectée par l'indice foliaire (LAI), tandis qu'elle ne l'est pas par le SAI. Pour rendre compte de la variation de l'effet du LAI sur la transpiration pendant qu'il varie de 0 à 4 au maximum, le modèle utilise une fonction quadratique pour calculer un coefficient réducteur (LAIF) :

$$
\mathrm{LAIF}=1-(\mathrm{LAI} / 4-1) * 2
$$

ce qui revient à dire que les premières tranches de LAI exercent plus d'effet que les dernières sur la transpiration.

Ce coefficient LAIF est utilisé pour calculer l'ETR par :

$$
\mathrm{ETR}=\mathrm{ETP} * \mathrm{LAIF}
$$

ou bien par :

$$
\mathrm{ETR}=\theta_{\text {aisponilhe }} * \mathrm{LAIF} / \mathrm{CT}
$$

suivant que l'une ou l'autre des conditions (16) ou (17) est réalisée.

L'évaporation de l'eau à partir du sol pourrait être considérée comme une interception par la litière et les horizons organo-minéraux superficiels. Il semble plus aisé de considérer ces horizons comme un compartiment séparé (fig. 2) d'où l'eau peut s'évaporer directement ou percoler vers le compartiment inférieur. La profondeur de ces horizons est fixée à $50 \mathrm{~mm}$ et ne varie pas. On ajoute l'eau à ce compartiment jusqu'à ce qu'il soit porté à la capacité en champ définie, comme il a été dit plus haut, par une conductivité hydraulique de $2 \mathrm{~mm} \cdot$ jour $^{-1}$.

Comme nous l'avons dit ci-dessus, la régulation de l'évaporation du sol superficiel se fait sur le même mode que pour la transpiration : soit en considérant que l'évapo- 
ration réelle (ER) est égale à l'évaporation potentielle (EP) si la teneur en eau est suffisante, soit, par une fonction linéaire de cette teneur. En outre, cette évaporation est réduite en fonction de l'indice foliaire (LAI) et de l'indice de tronc (SAI) de manière similaire à ce qui a été fait pour définir l'effet du LAI sur la transpiration. Un paramètre (CE) décrivant la disponibilité de l'eau de la litière pour l'évaporation est introduit dans le calcul d'une façon parallèle à ce qui a été fait pour le calcul de l'ETR.

L'empirisme des relations énumérées ci-dessus est évident. Il traduit le nombre très limité d'intrants requis par le modèle en comparaison avec ce que des modèles plus complexes exigent. Toutefois, si les valeurs de ces paramètres ont été fixées par une validation suffisamment étendue et que, comme nous le montrons, ces valeurs sont d'application générale, cette approche se justifie amplement.

\section{Résultats et discussions}

a) Les intrants utilisés pour le calcul du débit en 1984 sont reproduits aux figures 5 et 6 . Rappelons que seules la température moyenne journalière et les hauteurs des précipitations sont requises. Les paramètres utilisés pour le calcul sont ceux qui figurent au tableau 2 qui également compare ces derniers aux valeurs résultant de longues séries d'observations dans le New Hampshire et la Caroline du Nord (cf. 2.D).

b) Nous avons repris à la figure 7 les données observées quotidiennement pour les débits mesurés et calculés pour 3 saisons de 1984 .

L'accord entre ces deux séries de valeurs est aussi bon qu'on pouvait l'espérer, compte tenu de ce qu'il est généralement possible d'obtenir dans ce type de simulation. On voit que le débit calculé suit fidèlement le débit mesuré avec une surestimation manifeste au printemps et en automne et une sous-estimation pendant les crues de mai et juin.

Mais on peut dire aussi que la période durant laquelle le débit calculé est inférieur au débit mesuré correspond à la saison de végétation, c'est-à-dire au laps de temps pour lequel on tient compte d'un indice foliaire. Nous reviendrons plus loin sur ce point.

Par ailleurs, toujours en saison estivale, il ressort que lajustement est bien meilleur pour les périodes sèches que pour les périodes pluvieuses. Ce qui suggère que le modèle semble être particulièrement bien calibré pour répondre aux précipitations occasionnelles de courte durée ; ce qui devrait correspondre à un seuil d'égouttement relativement élevé. Mais, le maintien du même seuil d'égouttement pour les pluies de forte intensité et de longue durée induit automatiquement une surestimation des pluies interceptées par le couvert et donc de l'évaporation; ce qui se traduit par une sous-estimation du débit. Le modèle mis au point pour le climat du New Hampshire ne serait donc pas directement transposable à notre climat océanique capricieux.

c) La comparaison entre les valeurs mesurées et simulées peut se faire de manière plus objective en utilisant l'une ou l'autre des méthodes statistiques qui ont été mises au point pour la comparaison des séries temporelles. 


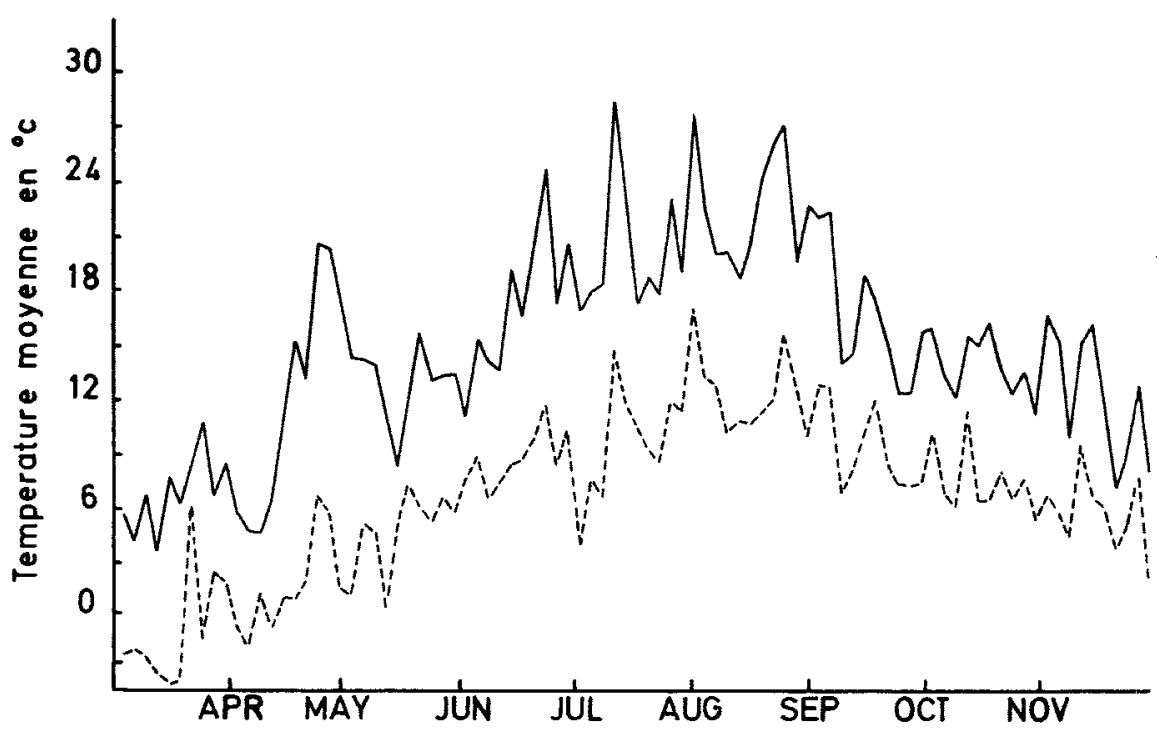

FIG. 5

Evolution en 1984 des températures maxima (-) et minima (_. -) à Chimay. Moyennes des observations par période de trois jours.

Patterns of maximum (__ and minimum (- .-) temperatures for 1984 in Chimay. Running mean values for three days periods.

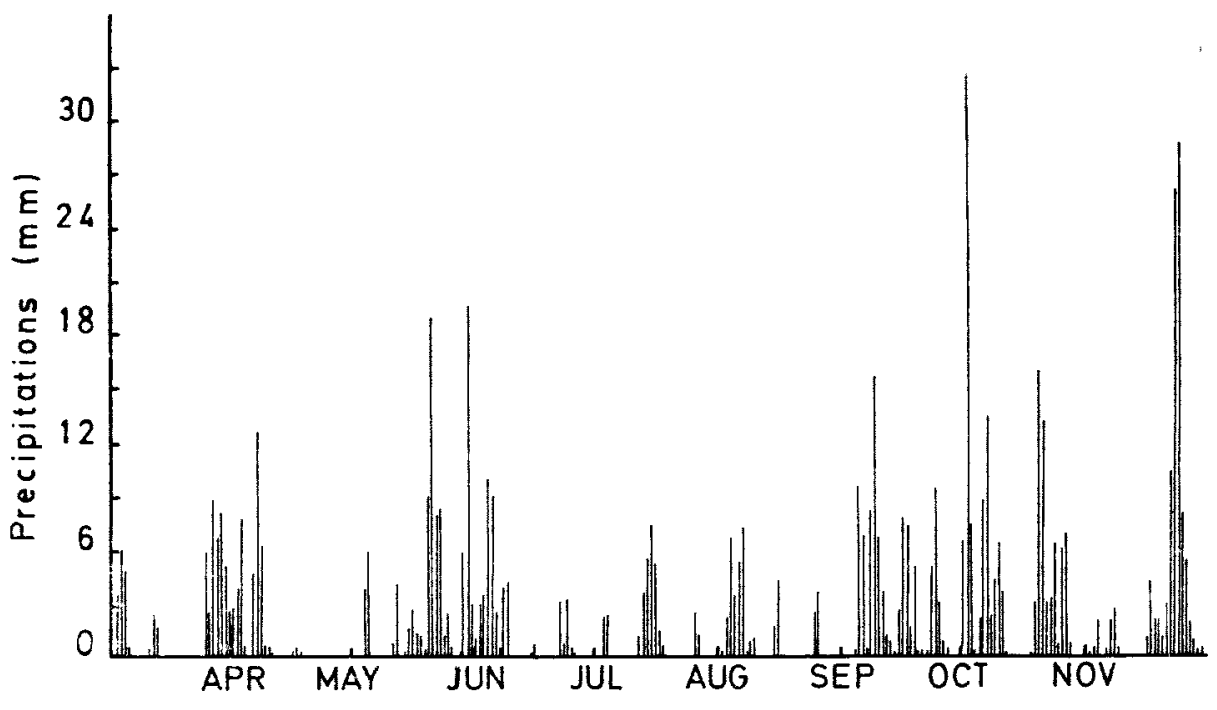

FIG. 6

Evolution en 1984 des précipitations journalières à Chimay. Patterns of daily rainfall for 1984 in Chimay. 

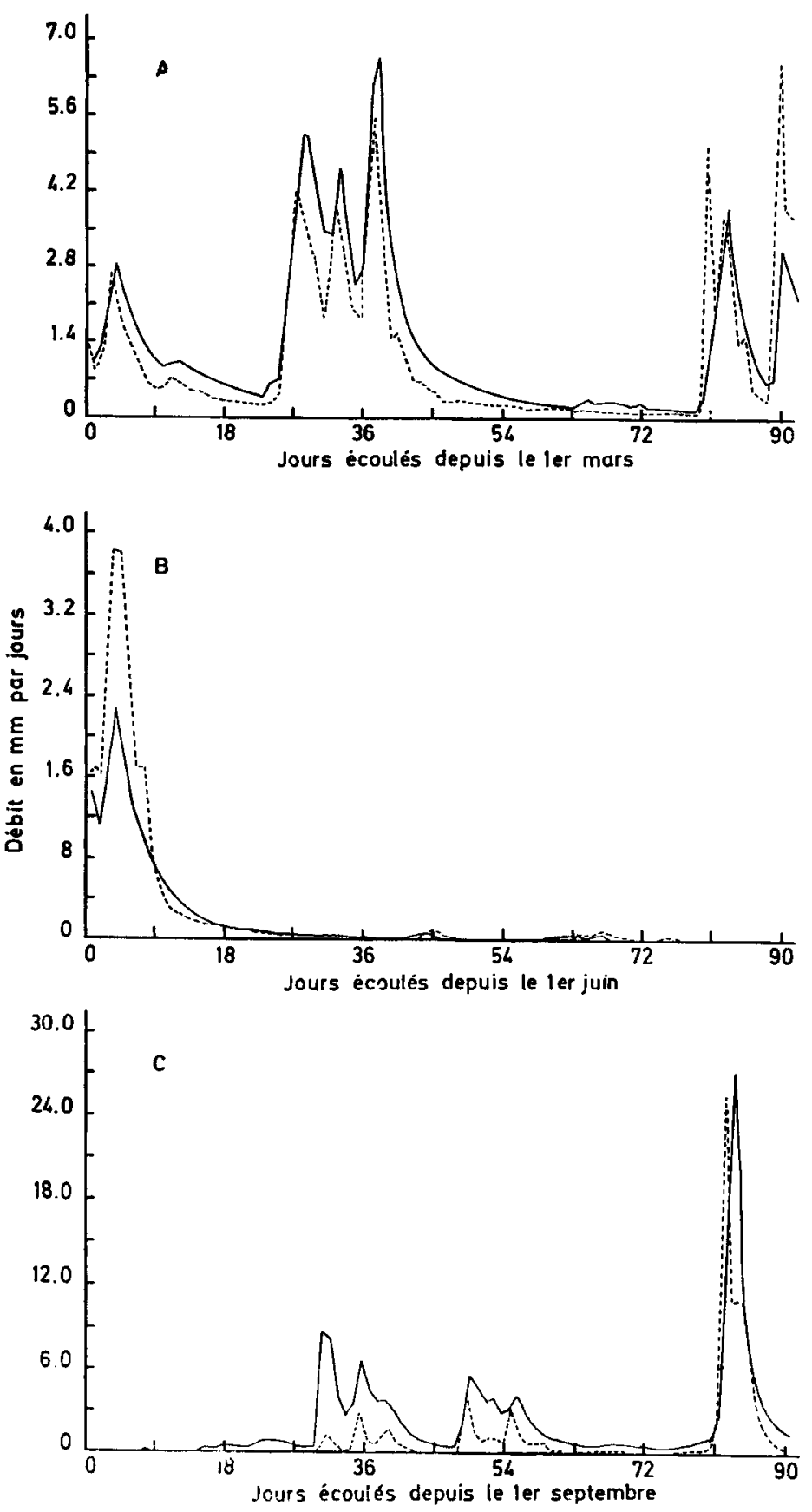
En effet, la comparaison qualitative des hydrogrammes telle qu'on peut la faire en examinant la figure 7 ne permet pas de se faire une idée précise de la valeur du calcul ni surtout de comparer la validation d'un modèle hydrologique s'étendant sur trois saisons seulement avec ce qui a été obtenu ailleurs pour des cycles beaucoup plus longs. Mais le calcul d'un simple coefficient de corrélation entre les deux séries temporelles est ouvert à diverses objections qui ont été discutées par McCueN \& SNYDER (1975). Aussi avons nous calculé les coefficients de corrélation ordinaire et amélioré suivant McCuen \& SNYDER (1975) les résultats présentés au tableau 3 se rapportant à une série de 9 mois et comparant deux stations américaines à Chimay. Il est intéressant de constater que, bien que les paramètres introduits dans le modèle n'aient en général subi aucune modification ad hoc, les coefficients de corrélation sont, dans l'ensemble, aussi bons à Chimay que dans les bassins versants américains et, il faut l'ajouter, parfois aussi mauvais.

d) Rappelons que les quelques paramètres modifiés l'ont été en fonction des observations et uniquement lorsque la chose s'avérait, à première vue, être indispensable. C'est ainsi, par exemple, que l'épaisseur de l'horizon exploité par le système racinaire forestier dans la Fagne de Chimay avait été estimé à $31 \mathrm{~cm}$ en moyenne (tabl. 2). Mais, on peut se demander si cette modification a une grande importance sur les résultats fournis par le modèle et légitime le grand nombre de mesures à effectuer sur le terrain de façon à obtenir aussi exactement que possible les valeurs nécessaires.

Deux possibilités s'offrent à nouveau pour répondre à cette question : soit la comparaison visuelle des résultats de simulation couvrant une fourchette de valeurs plausibles pour le paramètre en question, soit, l'analyse statistique de corrélation entre les hydrographes observés et simulés.

C'est ce que montre la figure 8 où sont présentés pour deux saisons les hydrogrammes simulés pour trois valeurs de la profondeur de la zone racinaire. Il est clair que ce paramètre exerce son influence en augmentant la crue lorsqu'il décroît et en diminuant le coefficient de récession lorsqu'il croît. Sans calcul, l'estimation quantitative de l'effet du paramètre montre une variation nette avec la saison. Mais, cette estimation quantitative suffit déjà aussi pour rejeter l'intérêt d'un ajustement ad hoc de ce paramètre. En effet, si on diminuait arbitrairement sa valeur de façon à éviter l'écart entre les valeurs simulées et mesurées au printemps et en automne, ce serait au prix d'une récession plus rapide et ce qui serait gagné pendant la crue serait perdu par la suite.

FIG. 7

Comparaison des débits calculés (-) et mesurés (-..-) dans le bassin versant de Chimay en 1984.

Le calcul est effectué avec les valeurs des paramètres repris au tableau 2.

Comparison of calculated (_-) and measured (- - ) flows of Chimay catchment for 1984.

The simulation is made with the parameter values listed in table 2.

A : Printemps - Spring.

B : Eté - Summer.

C : Automne - Autumn. 
TABLEAU 3

Comparaison des coefficients de corrélation ordinaire $(r)$ et amélioré suivant MCCUEN et SNYDER (R) entre les hydrographes mesurés et calculés. pour trois sites (en Caroline du Nord, dans le New Hampshire et à Chimay), sans modification des paramètres du modèle.

Comparison of the usual $(r)$ and improved correlation coefficients according to MCCUEN and SNYDER $(R)$ between measured and calculated hydrographs for three sites (North Carolina, New Hampshire and Chimay) without modification of the model parameters.

\begin{tabular}{|c|c|c|c|c|c|c|}
\hline & \multicolumn{2}{|c|}{ Coweeta } & \multicolumn{2}{|c|}{ Hubbard Brook } & \multicolumn{2}{|c|}{ Chimay } \\
\hline & $\mathrm{r}$ & $\mathrm{R}$ & $r$ & $\mathbf{R}$ & $\mathrm{r}$ & $\mathbf{R}$ \\
\hline Mars & 0,673 & 0,64 & 0,968 & 0,88 & 0,986 & 0,68 \\
\hline Avril & 0,901 & 0,73 & 0,669 & 0,46 & 0,986 & 0,76 \\
\hline Mai . & 0,978 & 0.50 & 0.474 & 0,19 & 0,934 & 0,82 \\
\hline Juin & 0944 & 0,64 & 0943 & 0,57 & 0,990 & 0,60 \\
\hline Juillet & 0,893 & 0,51 & 0,758 & 0,73 & 0,361 & 0,24 \\
\hline Août & 0,977 & 0,25 & 0,942 & 0,69 & 0,864 & $0,5 \mathrm{I}$ \\
\hline Septembre .. & 0,864 & 0,29 & 0,910 & 0,78 & 0,900 & 0,09 \\
\hline Octobre. & 0,625 & 0,31 & 0982 & 0,88 & 0,566 & 0,15 \\
\hline Novembre .. & 0,781 & 0,59 & 0,988 & 0,76 & 0,911 & 0,60 \\
\hline
\end{tabular}

La comparaison quantitative de l'effet du paramètre sur la qualité de la simulation peut également se faire par l'examen des coefficients de corrélation. C'est ce que montre le tableau 4 où les coefficients de corrélation sont reproduits pour 3 valeurs du paramètre. Il se confirme clairement qu'il n'y a aucun intérêt à s'écarter de la valeur initialement choisie, même si l'accord trouvé entre les hydrographes calculés et simulés pour un mois donné peut se trouver amélioré.

e) Il serait fastidieux de reproduire ici toutes les comparaisons qui ont été faites sur l'influence des paramètres « ajustables» du tableau 2. Mais, en raison de son intérêt intrinsèque et de la possibilité d'une valeur spécifique à déterminer pour l'écosystème qui nous intéresse, nous reproduisons à la figure 9 l'effet du coefficient d'interception (INC) des précipitations par le couvert forestier sur le débit calculé.

On voit, comme il fallait s'y attendre, que des variations de ce paramètre n'ont d'effets que si le LAI et la température atteignent des valeurs suffisantes. C'est le cas, pour 1984, de fin mai à octobre : c'est un coefficient d'interception de 0,50 contre 0,75 initialement prévu, qui permet de s'approcher le plus du débit mesuré. En relation avec ce qui a déjà été dit plus haut, on peut penser que lá constance du paramètre caractérisant l'interception amène une surestimation de l'évaporation au cours de la saison de végétation.

Si l'un ou l'autre de ces facteurs n'atteint pas une valeur critique, le coefficient d'interception choisi est sans importance dans les limites de 0,5 à 1,0 . 


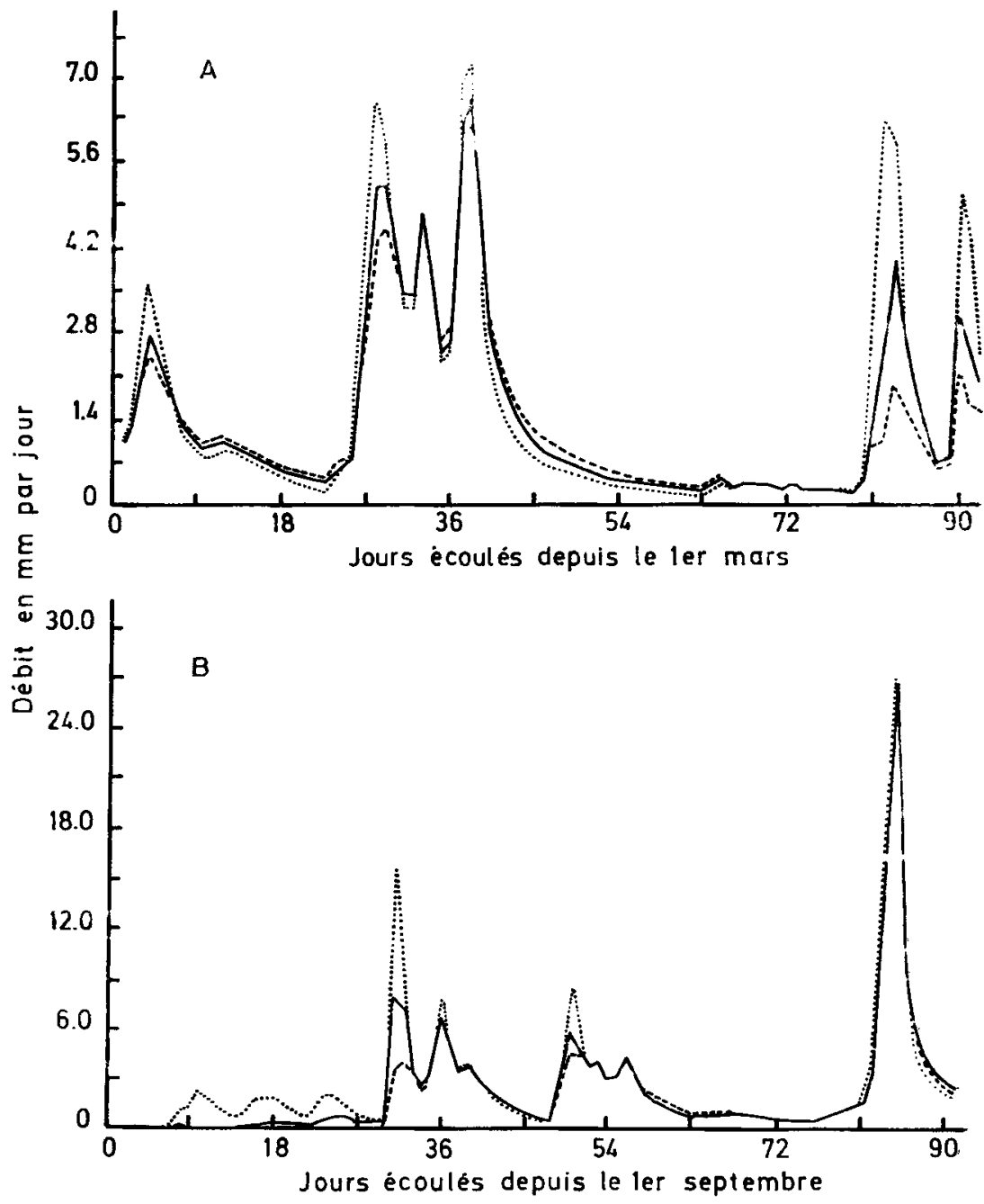

FIG. 8

Effet de la profondeur de la zone racinaire (EZDFP) sur le débit calculé du bussin versant de Chimay en 1984.

Effect of root zone depth on the calculated flow of Chimay catchment in 1984.

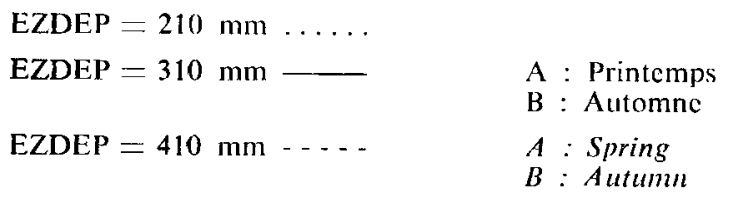



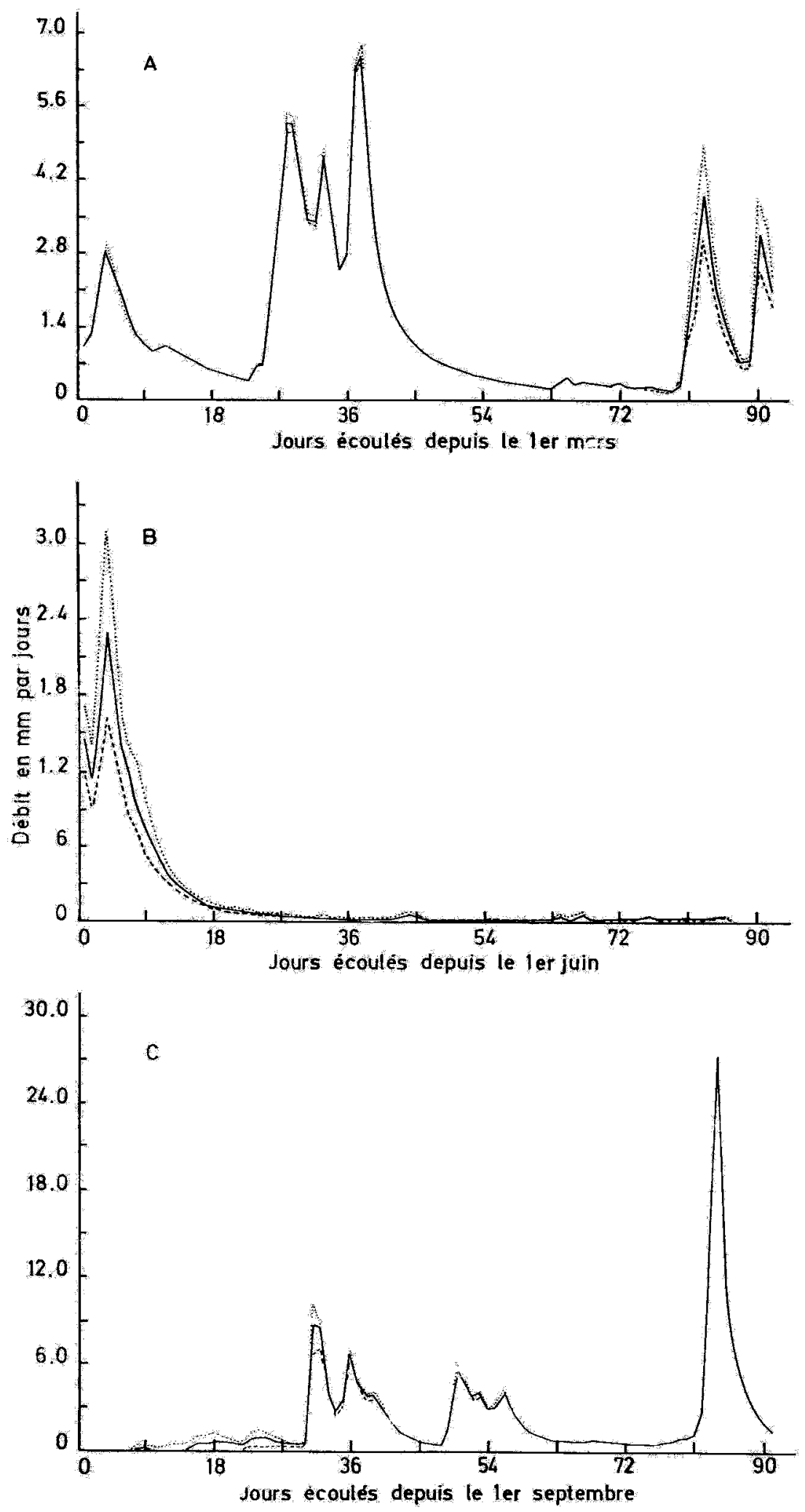


\section{TABleav 4}

Effet de la profondeur de la zone racinaire sur les coefficients de corrélation ordinaire ( $r$ ) et amélioré suivant MCCUEN et SNYDER $(R)$ entre les valeurs journalières de l'hydrographe calculé et simulé, pour le bassin versant de Chimay.

Effect of root zones depth on the usual $(r)$ and ameliorated according to MCCUEN and SNYDER $(R)$ correlation coefficients between measured and calculated daily values of the hydrographs for the Chimay catchment.

\begin{tabular}{|c|c|c|c|c|c|c|}
\hline & \multicolumn{2}{|c|}{$20 \mathrm{~cm}$} & \multicolumn{2}{|c|}{$30 \mathrm{~cm}$} & \multicolumn{2}{|c|}{$55 \mathrm{~cm}$} \\
\hline & $\mathbf{r}$ & $\mathbf{R}$ & r & $\mathbf{R}$ & r & $\mathbf{R}$ \\
\hline Mars & 0,580 & 0,20 & 0,986 & 0,68 & 0,523 & 0,11 \\
\hline Avril & 0,992 & 0,76 & 0,986 & 0,76 & 0,684 & 0,48 \\
\hline Mai . & 0,919 & 0,74 & 0,934 & 0,82 & 0,907 & 0,32 \\
\hline Juin & 0,985 & 0,74 & 0,990 & 0,60 & 0,991 & 0,39 \\
\hline Juillet & 0,339 & 0,18 & 0,361 & 0,24 & 0,425 & 0,36 \\
\hline Août $\ldots \ldots$ & 0,847 & 0,45 & 0,864 & 0,51 & 0,851 & 0.58 \\
\hline Septembre .. & 0,909 & 0,04 & 0,900 & 0,09 & 0,658 & 0,54 \\
\hline Octobre .... & 0,551 & 0,14 & 0,566 & 0,15 & 0,878 & 0,31 \\
\hline Novembre .. & 0,886 & 0,51 & 0,911 & 0,60 & 0,849 & 0,56 \\
\hline Ensemble .. & 0,719 & 0,61 & 0,821 & 0,68 & 0,810 & 0,59 \\
\hline
\end{tabular}

Les prévisions du modèle peuvent être appréciées d'une façon beaucoup plus globale en comparant le résultat du calcul pour l'évapotranspiration annuelle moyenne avec ce qui a été mesuré ailleurs où ce qui peut être calculé par des formules globales plus simples comme celle de Turc $(1951,1954)$ ou celle de Thornthwaite (1948). Bien entendu ,nos calculs ne couvrent qu'un très petit nombre d'années par comparaison à ce qui a été fait ailleurs. Nous calculons pour une pluviométrie totale de $1999 \mathrm{~mm}$ pour la période du $1^{\text {*r }}$ juillet 1981 au 31 août 1983 une évaporation totale de $794 \mathrm{~mm}$, soit $40 \%$ des précipitations. Cette valeur est remarquablement proche de celle qui est citée par LiKEns et al. (1967) pour la forêt du Hubbard Brook où l'on a

F1G. 9

Effet du coefficient dinterception (INC)

sur le débit caclulé du bassin versant de Chimay en 1984.

Effect of interception coefficient on the calculated flow of Chimay catchment in 1984.

$$
\begin{aligned}
& \text { INC }=0,50 \ldots \ldots \quad A: \text { Printemps } \\
& \text { INC }=0,75-B: \text { Eté }
\end{aligned}
$$

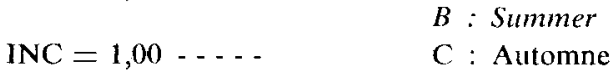

$$
\begin{aligned}
& \text { C : Autumn }
\end{aligned}
$$


mesuré une évapotranspiration équivalente à $42 \%$ des précipitations. D'autre part, Liebscher (1974) a observé dans les forêts du Harz une évaporation moyenne de $480 \mathrm{~mm}$ sur une précipitation de $1265 \mathrm{~mm}$, soit 38 p. 100 pour une période de 15 ans.

L'application de formules globales comme celle de TuRc aux années 1981 à 1983 donne une évaporation calculée de 53 p. 100 au lieu des 40 p. 100 observés. L'E.T.P. calculée suivant Thornthwarte donne une valeur plus élevée encore puisque l'approvisionnement et la disponibilité de l'eau ne sont pas prises en compte, soit $506 \mathrm{~mm}$.

\section{Conclusion}

La conclusion la plus intéressante que l'on puisse tirer de cet essai d'adaptation du modèle est, d'une part, le caractère assez général des valeurs des paramètres choisis et, d'autre part, la relative insensibilité du modèle à des fluctuations parfois importantes de ces paramètres. En ce qui concerne ce dernier point, il est tentant de penser que la stabilité du modèle est due à ce que sa structure reflète suffisamment bien celle de l'écosystème y compris sa stabilité intrinsèque.

Le caractère général des paramètres figurant dans les équations décrivant le mouvement de l'eau du sol est très probablement dû à ce que les propriétés imprimées aux couches superficielles du profil par la végétation forestière prennent le pas sur celles qui résultent du développement de la partie minérale du profil. C'est là probablement l'une des différences essentielles entre les modèles hydrologiques forestiers et agricoles.

La validation des calculs effectués grâce à ce modèle par le biais du débit d'un bassin versant observé durant quatre ans peut sembler insuffisante.

Il ne serait guère raisonnable d'espérer pouvoir valider le modèle en comparant ses prévisions de sécheresse édaphique aux observations faites sur le sol. L'hétérogénéité des précipitations sous forêt est telle qu'une estimation de la teneur en eau du sol demanderait un nombre de sondages prohibitifs. Il n'en est pas de même si le sol forestier se ressuie rapidement en raison de sa texture. C'est ce qui explique que Halldin et al. (1985) ont pu utiliser cette mesure en forêt de Fontainebleau.

Par contre, il semblerait intéressant à l'avenir de moduler l'interception en tenant compte des seuils d'égouttements, dont l'effet est bien plus important en climat océanique que dans l'est des U.S.A., et en introduisant un paramètre traduisant les variations de l'effet respectif du LAI et du SAI au cours des saisons de façon plus précise et plus réaliste que ce qui a été fait par l'équation (18).

Recu le 4 novembre 1985.

Accepté le 10 mars 1986. 


\section{Remerciements}

Un travail de ce genre repose sur la patience et la bonne volonté d'observateurs qui, jour après jour, enregistrent les données nécessaires.

Il nous est particulièrement agréable de remercier nos collaborateurs bénévoles : Pierre Eris, Jean-Rémy Nicolas, Micheline Laudelout et surtout Joseph Ori.Éans de lia Station météorologique de Sivry dont les données forment notre base de référence. Nous remercions également le personnel du Laboratoire forestier et spécialement Cécile TiLquiN et Monique Pifrrard.

We are also deeply indebted to $\mathrm{D}^{\mathrm{r}}$ A. FEDERER who provided us with detailed information about his experience on the use of the model. One of us (H.L.) is especially thankful to him for a thorough visit of the Hubbard Brook Forest Laboratory.

\section{Summary}

\section{Application of an hydrological model to a forest catchment of Wallonie}

In spite of the fact that a plethora of hydrological models have been described, at limited number only can be found which can be applied to forested catchments. Among those, several require input of data which are available only if well equiped ecoclimatological stations are present within or near the catchment studied. Since as rule, the data available for input are limited to maximum and minimum temperatures and rainfall, we have tried to adapt an existing model developped by FEDERER et al. for the forests of the North Eastern United States. Although this model is deterministic in nature, it is not very demanding with respect to the amount of input. Due to its deterministic nature, it seemed susceptible of being easily adapted to the conditions of forested catchments in North-Western Europe.

The study was carried out on a forested catchment located in the Fagne de Chimay in the immediate vicinity of the Forest Experimental Station of this University, about $200 \mathrm{~km}$ N.E. of Paris. The area of the catchment was about 1000 ha of which $95 \mathrm{p}$. 100 was under an oakhornbeam forest resting on a heavy clay soil derived from the weathering of Fammennian shale. The input data were the daily minimum and naximum temperatures and the precipitations measured at 6 points within or in the immediate vicinity of the catchment. For validating the results of the model, the streamflow was measured at a weir located at the outlet of a 400 ha subcatchment. No ad hoc adjustment of the parameter of the model was carried out with the exception of those which obviously had to be changed such as latitude, slope, aspect, soil depth, dates of leafling and leaf fall, rain snow separation temperature. Other parameters were left as they had been adjusted by Fi:Dirik in two studies in the North and South Eastern United States (New Hampshire and North Carolina). A comparison of the parameters is presented in Table... Where it can be seen that in some cases, the numcrical values were rounded or the average between the two U.S. values was taken or for obvious reasons of similitude the New Hampshire value was used when the differences between the two American values were too large. Shortly no ad hoc adjustment was carried out.

It is shown that the predicted values of the streamflow did agree fairly well with the observed flow and that the statistics of the calculated an experimental flow rates were as good as those observed in the United States where the calibration of the model had been obtained from observations on a much longer temporal basis.

It seems likely that the forest vegetation plays a prominent role with respect to hillslope hydrological relations by creating and maintaining organic layers above the minerat horizons especially when the latter are imprevious.

Simulation studies with the model demonstrated the necessity of a better knowledge of leaf area values and of their seasonal changes in North Western European forests. 


\section{Références bibliographiques}

Aussfenac G., 1968. Interception des précipitations par le couvert forestier. Ann. Sci. For., 25, $135-156$.

Aussenac G., Boulangeat C., 1980. Interception des précipitations et évapotranspiration réelle dans des peuplements de feuillus (Fagus sylvatica) et de résincux (Pseudotsuga menziesii). Ann. Sci. For., 27, 91-107.

BEALI H.W., 1934. The penetration of rainfall through hardwood and softwood forest canopy. Ecology, 15, 412-415.

Bodeux A., 1954. Recherches écologiques sur le bilan d'eau sous la forêt et la lande de Haute Campine. Agricultura, 2, i-80.

Brechtel H., 1969. Wald und abfluss. Methoden zur Erforschung der Bedeutung des Waldes für das Wasser angebot. Deutsche Gewasserkundl. Mitt. Sonderheft, 24-31.

Bultor F., Dupriez G.L., Bodeux A., 1982. Interception de la pluie par la végétation forestière. Estimation de l'interception journalière à l'aide d'un modèle mathématique. J. Hydrol., 17, 192-223.

Campbell G.S., 1974. A simple method for determining unsatured conductivity from moisture retention data. Soil. Sci., 117, 311-314.

Christophersen N., Rustad S., Steip H.M., 1984. Modelling streamwater chemistry with snow melt. Phil. Trans. R. Soc. London B., 305, 427-439.

Christophersen N., Wright R.F., 1981. Sulfate budget and a model for sulfate concentrations in streamwater at Birkenes, a small forested catchment in Southernmost Norway. Water Resour. Res., 17, 377-389.

Eidman F., 1959. Die Interception in Buchen- und Fichtenbeständen. Ergebnis mehrjähriger Untersuchungen im Rothaargebirge (Sauerland). Ass. Int. Hydr. Scient. Public, Gentbrugge, $40 \mathrm{p}$.

FEDERER C.A., 1973. Forest transpiration greatly speeds streamflow recession. Water Resour. Res., 9, 1599-1604.

FEDERER C.A., 1979. A soil-plant-atmosphere model for transpiration and availability of soil water. Water Resour. Res., 15, 555-562.

Federer C.A., 1982. Transpirational supply and demand : plant soil and atmospheric effects evaluated by simulation. Water Resour. Res., 18, 355-362.

Federer C.A., Lash D., 1978. Brook : a hydrologic simulation model for Eastern forests. Water Resour. Res. Center, University of New Hampshire, Durham, New Hampshire, Res. Rep. nr., 12.

GRIP H., 1982. Water chemistry and runoff in forest streams at Kloten. Uppsala University, U.N.G.I. Report nr. 58, 144 p.

Halldin S., Saugier B., Pontailler J.Y., 1985. Evapotranspiration of a deciduous forest : simulation using routine meteorological data. J. Hydrology, 75, 323-341.

Hamon W.R., 1963. Computation of direct runoff amounts from storm rainfall. Int. Assoc. Sci. Hydrol. Publ., 63, 52-62.

Helvey J.D., PATRIC J.H., 1965. Canopy and litter interception of rainfall by hardwoods of Eastern United States. Water Resour. Res., 1, 193-206.

Hewlett J.P., Hibbert A.R., 1967. Factors affecting the response of small watersheds to precipitation in humid area. In : Forest Hydrology, Ed. Sopper W.E. and Lull H.W., Pergamon Press, London, 275-290.

HoRTon R.E., 1919. Rainfall interception. Monthly Weather Review, 47, 603-623.

Horton R.E., 1937. Hydrologic interrelations of water and soils. Proc. Soil Sci. Soc. Am., $1,401-429$. 
LANDSBERG H.E., 1983. Variability of the precipitation process in time and space. In : Sampling and analysis of rain. Sally A. Campbell ed. ASTM Special Technical Publication, 823 p.

Legrand-Marce C., Laudelout H., 1985. Longitudinal dispersion in a forest stream. J. of Hydrology, 78, 317-324.

LEONARD R.E., 1961. Net precipitation in a Northern hardwood forest. J. Geophys. Res., 66, $2417-2121$.

LIEBSCHER H.-J., 1974. Forstlich-hydrologische Untersuchungen in bewaldeten Versuchsgebieten im Oberharz. IX Wasserhaushalt in den Oberharzer Versuchsgebieten, Aus dem Walde - Mitteilungen aus der Niedersächsischen Landestorstverwaltung, 22, 172-198.

Likens G.E., Bormann F.H., Johnson N.M., Pierce R.S., 1967. The calcium, magnesium, potassium and sodium budgets for a small forested ecosystem. Ecology, 48, 772-785.

LuNDQuist D., 1976. Simulation of the hydrologic cycle. SNSF Project, Oslo-As, Norway TR $23 / 76,28 \mathrm{p}$.

LUNDQUISr D., 1977. Hydrochemical modelling of drainage basins. SNSF Project, Osto-As, Norway IR 31/77 (en norvégien), $27 \mathrm{p}$.

LunDQuist D., 1978. Practical use of a simple conceptual model. Paper presented at the Nordic Hydrological Conference, National Board of Waters, Helsinki.

MCCUEN R.H., SNYDER W.M., 1975. A proposed index for comparing hydrographs. Water Resoutr. Res., 11, 1021-1024.

MrTchell, 1930. Interception of rainfall by the forest. J. Forestry, 28, 101-102.

Mrtscherliscu G., Mor.t. W., 1970. Untersuchungen über die Niederschlags- und Boden Feuchtigkeitsverhältnisse in einigen Nadel und Laubholzbeständen in der Nähe von Freiburg. Alg. Forst. u. J. Zig., 141, 49-60.

Molchanov A.A., 1971. Cycles of atmospheric precipitation in different types of forests of natural zones of the U.S.S.R. In : Productivity of Forest Ecosystems, Ed. P. Duvigneaud, Unesco, Paris, 707 p., 49-68.

Montuore A., Cajot O., 1983. Aspects hydrologiques des bassins expérimentaux de la Robinette et du Waroneu (Hautes-Fagnes, Belgique). In : Les écosystèmes forestiers en Wallonie, Hanotiaux G. ed., Fac. Sci. Agr., Gembloux, 137 p., 57-69.

Noirfalise A., 1959. Sur l'interception de la pluie par le couvert dans quelques forêts belges. Bull. Soc. R. For. Belgique, 66, 433-439.

NoIrfalis: A., 1975. Conséquences de la monoculture des conifères pour la conservation des sols et pour le bilan hydrologique. Notes techniques Centre Ecologic Forestière, Gembloux, $\mathrm{n}^{\circ 24}, 34 \mathrm{p}$.

OVINGron J.D., 1954. A comparison of rainfall in different woodland. Forestry, 27, 41-53.

Poncelet L., Martin H., 1947. Esquisse climatologique de la Belgique. Mémoire Institut Royal Météorologique de Belgique, 27, 625 p.

Remy J., 1980. Carte des sols de la Belgique : planchette de Seloignes 190 E. Centre de Cartographie des sols de la Belgique Méridionale, Gembloux.

REMY J., 1981. Carte des sols de la Belgique : planchette de Rance 181 H. Centre de Cartographic des sols de la Belgique Méridionale, Gembloux.

SChNock G., 1967. Recherches sur l'écosystème forêt, série B. La chênaie mélangée calcicole de Virclles-Blaimont. Contribution $\mathrm{n}^{*} 17$ : réception des précipitations et écoulement le long des troncs en 1966, Bull. Inst. Sci. Nat. Belg., 43, 1-15.

Schnock G., Galoux A., 1967. Recherches sur l'écosystème forêt, série B. La chênaie mélangée calcicole de Virelles-Blaimont. Contribution $n^{\circ} 8$ : réception des précipitations et égouttement, Bull. Inst. Sci. Nat. Belg., 43, 1-30.

Sougnez N., 1967. Les forêts de la Lorraine belge. Presses Agronomiques, Gembloux, $114 \mathrm{p}$.

Sougnez N., 1978. Les chênaies-charmaies du district calcaire mosan. Communications du Centre d'écologie forestière et rurale, Gembloux, Nouvelle série n" 23,85 p. 
SWIFT L.W., 1976. Algorithm for solar radiation on mountain slopes. Water Resour. Res., 12, $108-112$.

Thornthwaite C.W., 1948. An approach towards a rational classification of climate. Georg. Rev., 38, 53.

Turc I., 1951. Nouvelle formule pour le calcul du bilan de l'eau en fonction des valelurs annuelles moyennes des précipitations et de la température. C.R., 233, 633.

Turc L., 1954. Calcul du bilan de l'eau : évaluation en fonction des précipitations et des températures, Association Internationale d'Hydrologic, Assemblée Générale de Rome, 3 (37), 188-202.

VAN Der PLOEG R.R., 1978. Entwicklung zweidimensionaler Modelle für den Wasserumsatz in Boden hängiger Fichtenstandorte des Harzes, Habilitationsschrift. Georg-August Universität Göttingen.

Whittaker R.H., Bormann F.H., Likens G.E., Siccama T.G., 1974. The Hubbard Brook Ecosystem Study : forest biomass and production. Ecol. Monogr., 44, 233-254.

Whitraker R.H., Woodwell G.M., 1967. Surface area relations of woody plants and forest communities. Am. J. Bot., 54, 931-939.

WRIGHT R.F., 1982. Norwegian models for surface water chemistry : an overview, Chap. 4 (73-87). In : Modclling of total acid precipitation impacts, Ed. Schnoor J. (ed.), Proc. Am. Chem. Soc. Meeting Las Vegas, Ann. Arbor. Science. 